Meta

Journal des traducteurs

Translators' Journal

\title{
La recherche sur les processus traductionnels et la formation en interprétation de conférence
}

\section{Daniel Gile}

Volume 50, numéro 2, avril 2005

Processus et cheminements en traduction et interprétation Processes and Pathways in Translation and Interpretation

URI : https://id.erudit.org/iderudit/011013ar

DOI : https://doi.org/10.7202/011013ar

Aller au sommaire du numéro

Éditeur(s)

Les Presses de l'Université de Montréal

ISSN

0026-0452 (imprimé)

1492-1421 (numérique)

Découvrir la revue

Citer cet article

Gile, D. (2005). La recherche sur les processus traductionnels et la formation en interprétation de conférence. Meta, 50(2), 713-726.

https://doi.org/10.7202/011013ar
Résumé de l'article

La recherche sur les processus cognitifs accompagnant l'interprétation de conférence est largement fondée sur des théories conçues en psychologie cognitive qu'il est difficile de valider par des procédures empiriques, notamment en raison de la complexité des processus et de la variabilité qui en résulte sur le plan holistique de la prestation. Les ressources institutionnelles et humaines de la communauté traductologique sont insuffisantes pour espérer un changement radical et de nombreuses applications didactiques immédiates. En revanche, les concepts et modèles développés ont une intéressante valeur explicative. Une recherche sur les processus plus proche des actions concrètes, telle qu'elle est menée par les traductologues de l'écrit, pourrait avoir des retombées didactiques directes plus nombreuses. 


\title{
La recherche sur les processus traductionnels et la formation en interprétation de conférence
}

\author{
DANIEL GILE \\ Université Lyon 2, Lyon, France \\ daniel.gile@laposte.net
}

\begin{abstract}
RÉSUMÉ
La recherche sur les processus cognitifs accompagnant l'interprétation de conférence est largement fondée sur des théories conçues en psychologie cognitive qu'il est difficile de valider par des procédures empiriques, notamment en raison de la complexité des processus et de la variabilité qui en résulte sur le plan holistique de la prestation. Les ressources institutionnelles et humaines de la communauté traductologique sont insuffisantes pour espérer un changement radical et de nombreuses applications didactiques immédiates. En revanche, les concepts et modèles développés ont une intéressante valeur explicative. Une recherche sur les processus plus proche des actions concrètes, telle qu'elle est menée par les traductologues de l'écrit, pourrait avoir des retombées didactiques directes plus nombreuses.
\end{abstract}

\section{ABSTRACT}

Research on cognitive processes in conference interpreting is largely based on theories developed within cognitive psychology which are difficult to validate empirically, in particular due to the complexity of the processes involved and to the resulting variability at the holistic level of interpreting performance. Institutional and human resources within the interpreting research community are too limited to allow much hope for radical changes and immediate didactic applications, but the concepts and models developed so far have explanatory value. If process-oriented research into conference interpreting were redirected towards concrete interpreting actions, in a similar approach to that taken by translation researchers, more direct didactic applications could be hoped for.

\section{MOTS CLÉS/KEYWORDS}

Interprétation de conférence, processus, variabilité, applications didactiques, signal, bruit

\section{Introduction : processus observables et processus cognitifs}

Le terme processus désigne de manière générique le déroulement d'un phénomène. S'agissant d'actions humaines, il peut désigner une suite d'opérations physiques, par exemple le long d'une chaîne de montage. En traduction, on peut également parler d'une succession d'actions du même type, allant par exemple du décrochage du téléphone pour prendre une commande initiale jusqu'à l'envoi du produit au client, voire jusqu'à l'enregistrement du paiement reçu, en passant par la réception du texte de départ, sa lecture, le marquage de problèmes, la consultation de sources documentaires, la rédaction d'un premier texte, sa révision, etc. Il s'agit là de la dimension observable du processus de traduction. En revanche, les processus cognitifs, qui correspondent aux opérations mentales du traducteur ou interprète, ne sont pas directement observables. Le savoir du praticien en ce qui concerne les processus observables est dans l'ensemble assez complet et fiable dans son environnement local 
(celui dans lequel il travaille), bien qu’il ne corresponde pas nécessairement aux processus observables dans d'autres environnements (autres entreprises, autres secteurs du marché, autres pays, autres langues). La traductologie contemporaine s'intéresse peu à la partie observable des processus, et emploie généralement le mot processus dans une acception plus limitée, qui correspond aux seuls processus cognitifs. Par ailleurs, une partie des travaux parfois rapportés sous le chapeau processus dans des volumes collectifs et des revues portent non pas sur les processus mêmes, mais sur certains phénomènes traductionnels qui ont un rapport avec les processus mais n'en font pas vraiment partie. C'est par exemple le cas de certaines études sur l'effet des conditions de travail sur le stress et la qualité de travail des traducteurs et interprètes. Le volet processus prend alors une dimension très large et perd son caractère spécifique.

Dans le présent article, l'analyse porte essentiellement sur la recherche (au sens scientifique du terme - voir ci-dessous) sur les processus traductionnels au sens plus étroit. Plus précisément, elle part du constat maintes fois confirmé du désintérêt, voir de l'hostilité d'une grande partie des formateurs de traducteurs et d'interprètes à l'égard de la recherche, et s'interroge sur l'utilité de l'activité traductologique en matière de processus pour la formation.

\section{Connaissances premières, connaissances et idées structurées, connaissances et théories scientifiques}

En interprétation comme en traduction, au début était la pratique. La formation, puis la recherche, sont intervenues dans un deuxième et un troisième temps respectivement. Notons toutefois que la formation a une très longue histoire, alors que la recherche traductologique est récente.

La pratique est nécessairement fondée sur un savoir linguistique, un savoir thématique et un savoir social, auxquels s'ajoute progressivement un savoir-faire. Au fil de l'expérience du praticien, celui-ci acquiert notamment une certaine connaissance et des idées intuitives sur les processus cognitifs qui accompagnent la traduction, ou plutôt sur certains éléments liés à ces processus cognitifs. Ainsi, il peut se rendre compte que la traduction comporte une certaine « recherche mentale» de mots et de structures, car les mots idoines ne «viennent» pas spontanément, qu'il arrive que la langue de départ et la langue d'arrivée «se mélangent dans son esprit» et lui font faire des xénismes ou douter de la correction de son expression en langue d'arrivée, qu'en interprétation simultanée, il lui arrive d'oublier avant de pouvoir les énoncer des éléments d'information du discours de départ pourtant captés, qu'il lui arrive d'anticiper la fin d'une phrase que prononce un orateur, qu'il écoute son propre discours en parlant, puisqu'il lui arrive de faire des autocorrections, etc. À ce niveau de conscience, on peut parler de connaissances et idées premières.

Ces connaissances et idées s'organisent en schémas plus cohérents quand le praticien commence à y réfléchir, à les expliciter et à les structurer (voir Kussmaul 1995: 5; Fraser 2000 : 112), le plus souvent quand il devient formateur et éprouve le besoin de construire une plateforme cohérente pour les conseils qu'il prodiguera aux apprentis dont il a la charge. De nombreux formateurs de traducteurs et d'interprètes, peut-être la majorité d'entre eux, enseignent sur la base de ces connaissances et idées structurées. Dans de nombreux cas, elles restent d'ailleurs individuelles, dans la mesure où chaque formateur applique ses propres idées, sans les confronter à celles des autres. 
Dans d'autres cas, la structuration passe par une socialisation, qui peut être verticale (un formateur enseigne à un étudiant qui devient à son tour formateur et utilise les mêmes connaissances et idées) ou horizontale (des groupes de formateurs échangent leurs connaissances et idées, en adoptent certaines et en rejettent d'autres).

À un troisième niveau se situent les connaissances et théories scientifiques. Ce terme désigne ici des connaissances et idées qui font l'objet d'un traitement scientifique, à savoir une théorisation et des vérifications par des méthodes de recherche empiriques. La méthode scientifique, comme on l'a appelée, se caractérise notamment par une démarche systématique, prudente, collective et critique. Les connaissances sont documentées et systématiquement vérifiées, et les idées, hypothèses et théories sont régulièrement soumises à l'épreuve des faits, sur le terrain (dans la recherche naturaliste) et en laboratoire (dans la recherche expérimentale), et à des échanges analytiques contradictoires et critiques.

La recherche est donc systématiquement sceptique à l'égard de ses propres connaissances et théories, et considère en principe qu'à tout moment, celles-ci ne représentent qu'une étape et sont appelées à être corrigées et complétées. C'est l'une des caractéristiques qui la différencient de la démarche du formateur praticien, qui agit sur la base de postulats, même si, quand il prend sa casquette de chercheur, il remet ces postulats en cause. Une autre caractéristique de la recherche scientifique est sa tendance à aller toujours plus loin dans l'exploration du phénomène qu'elle étudie, essentiellement dans le sens de la profondeur. Le formateur, en revanche, a besoin de certaines connaissances et de certains principes pour orienter ses étudiants, mais n'a pas vocation à aller plus loin que ses besoins didactiques.

Ces différences expliquent peut-être le fait que de nombreux formateurs dans les domaines de la traduction et de l'interprétation, voire la majorité d'entre eux, se contentent des connaissances premières ou des connaissances et idées structurées acquises intuitivement, ou héritées d'autres formateurs dont les propres connaissances sont d'origine intuitive, et ne font pas appel à la recherche, que ce soit dans sa composante de théorisation ou dans sa composante empirique (rappelons que la théorie est la partie conceptuelle de la démarche scientifique, alors que les études empiriques, qu'elles soient naturalistes ou expérimentales, sont les explorations et vérifications factuelles permettant d'élaborer des hypothèses et théories et de les vérifier). D'autres formateurs considèrent que la recherche est indispensable, tant pour la compréhension de la pratique que pour son amélioration et pour l'enseignement.

Comme toute classification, cet ensemble de trois catégories d'idées et connaissances est une construction intellectuelle; nous l'espérons utile, mais elle ne saurait être considérée comme précise et absolue. Les trois catégories correspondent plutôt à des zones sur un continuum de complexité du traitement intellectuel des connaissances et idées (figure 1).

FIGURE 1

L'axe du traitement des connaissances et idées

\begin{tabular}{|c|c|c|}
\hline $\begin{array}{l}\text { Connaissances et } \\
\text { idées premières }\end{array}$ & $\begin{array}{l}\text { Connaissances et } \\
\text { idées structurées }\end{array}$ & $\begin{array}{l}\text { Connaissances et } \\
\text { théories scientifiques }\end{array}$ \\
\hline
\end{tabular}

On notera que les impressions premières "pures» ne sont pas directement accessibles à l'observateur, puisqu'elles commencent à se structurer dès qu'elles sont 
verbalisées, et a fortiori quand des praticiens échangent des connaissances et idées avec des collègues. Quand elles font l'objet de discussions contradictoires et critiques, elles se rapprochent des connaissances et théories scientifiques, mais n'atteignent ce statut pleinement que lorsque cet examen critique devient systématique et répond à certaines règles et normes scientifiques. Le présent article se concentre sur les connaissances structurées et les théories scientifiques telles qu'elles sont définies plus haut, et notamment sur ces dernières, pour en analyser l'intérêt et les limites au regard de la formation.

\section{La recherche sur les processus en interprétation}

Les premiers écrits témoignant d'une réflexion systématique sur les processus cognitifs sous-jacents à l'interprétation (de conférence) datent des années 1950. Dès les écrits de Herbert (1952) et Van Hoof (1962), on trouve des observations qui en apportent la preuve. Ainsi, ils considèrent tous les deux que la simultanée n'est qu'une consécutive accélérée, et Van Hoof postule une prise de décisions au centre de la consécutive, mais absente de la simultanée.

Le mémoire de M. A. de Eva Paneth (1957), premier texte universitaire sur l'interprétation par un interprète, se situe déjà dans les connaissances et idées scientifiques, même si son texte relève encore de l'introspection et paraît aujourd'hui méthodologiquement modeste. Les travaux et écrits des psychologues et psycholinguistes qui se sont penchés sur l'interprétation vers la fin des années 1960 et pendant les années 1970 (Oléron et Nanpon, Goldman-Eisler, Barik et surtout Gerver - voir Pöchhacker et Shlesinger 2002 et Pöchhacker 2004) se situent pleinement dans la démarche scientifique, en ce sens qu'ils s'intègrent dans un processus de débat contradictoire au sein de la communauté scientifique avec tentatives d'exploration des faits et vérification empirique d'hypothèses. En l'occurrence, les théories mises en œuvre sont celles de la psychologie cognitive et de la psycholinguistique, et la démarche empirique choisie est expérimentale. En 1976, Barbara Moser, jeune interprète tout juste diplômée de l'université d'Innsbruck, adapte un modèle de la compréhension du discours du psychologue Dominic Massaro pour construire un modèle du processus de l'interprétation simultanée (voir Moser, 1978). Vers le même moment, Danica Seleskovitch, interprète de conférence et surtout professeure d'interprétation, diffuse sa «théorie du sens», qui postule un processus de déverbalisation intermédiaire entre la compréhension du discours de départ et la reformulation du discours d'arrivée chez l'interprète (voir par exemple un extrait dans Pöchhacker et Shlesinger 2002 : 120-129.). Sans porter ici de jugement sur l'intérêt de cette idée, sur le continuum allant des impressions et connaissances premières aux connaissances et théories scientifiques, on peut la situer quelque part entre les idées structurées et les théories scientifiques. En effet, l'idée de déverbalisation entre la compréhension et la production est sans doute fondée sur une importante expérience personnelle, sur beaucoup de réflexion et sur l'observation, mais Seleskovitch ne l'a pas intégrée dans un débat collectif et contradictoire avec les théories et idées d'autres auteurs contemporains, ni essayé de la vérifier par des procédures rigoureuses, qu'elles soient naturalistes ou expérimentales. C'est en cela que son activité de théorisation correspondait à ce que j'ai appelé en 1990 personal theorising; l'expression mettait en exergue cet isolement 
choisi par elle et ses disciples, mais n'avait pas les autres sens et connotations que lui ont attribué par la suite de nombreux chercheurs qui l'ont citée.

À partir de la fin des années 1980, la communauté de la recherche sur l'interprétation s'est rapprochée à nouveau des normes de la recherche scientifique. En réalité, dès les années 1970, en Union Soviétique, l'ancien interprète à l'ONU Ghelly Chernov, qui travaillait avec des psycholinguistes, avait proposé son propre modèle du processus de l'interprétation simultanée, qui se focalisait sur un processus de "prévision probabiliste» (voir son article reproduit dans Pöchhacker et Shlesinger 2002). Par ailleurs, à Londres, Jennifer Mackintosh, une autre interprète, soutenait un mémoire de M. A. fondé sur les théories de traitement propositionnel du langage par Kintsch et Van Dijk (Mackintosh 1983). Toutefois, dans les pays occidentaux régnait la "théorie du sens», et les voix dissidentes avaient du mal à se faire entendre. Il a fallu attendre l'initiative triestine d'une importante conférence sur la didactique de l'interprétation (Gran et Dodds 1989) pour qu'elles résonnent au sein de la communauté. Depuis, les chercheurs en interprétation se sont dans leur grande majorité résolument tournés vers les normes de la recherche scientifique, en puisant des idées et théories dans les sciences cognitives. La neurolinguistique, la psychologie cognitive, la pragmatique sont très présentes dans la recherche sur les processus de l'interprétation (voir notamment Setton 1999, 2003).

Certaines caractéristiques de la recherche sur les processus en interprétation prennent du relief surtout quand on compare celle-ci à la recherche sur les processus en traduction écrite. Contrairement à la recherche sur l'interprétation, la recherche sur la traduction n'a commencé à se pencher sérieusement sur les processus qu'après une longue histoire de réflexion sur la fidélité, la traduisibilité et d'autres questions axées sur le produit. Depuis les premiers travaux de Krings et Lörscher dans les années 1980, le paradigme de recherche empirique sur le processus le plus répandu est celui des TAP (think-aloud protocols, ou verbalisation à voix haute), avec des variantes; plus récemment, il a notamment été complété par des procédures de suivi des opérations de traduction sur et par ordinateur (voir par exemple TirkkonenCondit et Jääskeläinen 2000; Hansen 1999; Hansen 2003). Comme le constate Fraser (2000: 119), la quasi-totalité de ces études sont des observations concrètes de ce que font les traducteurs, le classent, l'interprètent, parfois à travers des modèles (voir Hönig 1995; Snell-Hornby et coll. 1999: 160-178). Dans la quasi-totalité des cas, leur point de départ est un ensemble d'observations concrètes recueillies systématiquement. Dans la recherche sur l'interprétation de conférence, il existe bien quelques travaux du même type, notamment sur la prise de notes (Andres 2000, Dam 2004), mais l'essentiel des efforts entrepris relève de la démarche inverse: les auteurs conceptualisent d'abord, et vérifient ensuite - quand ils le peuvent (voir plus loin). La conceptualisation en question a le plus souvent comme base l'observation et l'introspection sur la réalité rencontrée sur le terrain par les auteurs praticiens (c'est le cas des modèles de D. Seleskovitch et de D. Gile) et, depuis le retour en force des sciences cognitives dans les années 1990, des théories psychologiques, linguistiques et neurolinguistiques. Il en résulte une différence frappante entre les modèles de la traduction et les modèles de l'interprétation les plus récents. Les premiers représentent des connaissances et des actions holistiques (stratégies, tactiques, lecture, écriture, consultation de sources), alors que les seconds s'articulent en majorité autour de modules cognitifs, d'éléments d'information et d'actions à un niveau bien plus détaillé et 
abstrait (systèmes de réception auditifs, structures sémantiques pré-linguistiques, exécutif central, boucle phonologique, calepin visuo-spatial, etc. - voir par exemple Mizuno, dans ce numéro spécial).

Quant à la recherche empirique en matière de processus, prépondérante en traduction, elle est très modeste en interprétation. Si l'on note depuis quelques années un certain nombre d'études qui rappellent les travaux des traductologues de l'écrit par leur démarche d'observation des processus (D. Andres a suivi le processus de prise de notes en consécutive, et P. Mead (2002) en a étudié le déroulement temporel du point de vue des pauses), la recherche empirique sur l'interprétation liée aux processus se concentre plutôt sur des indicateurs donnant indirectement des informations sur ces processus: réactions physiologiques telles que le stress, la tension artérielle ou la conductance de la peau, manière de traduire, nombre et types d'erreurs sous différentes conditions expérimentales, etc. À quelques exceptions près, ces études sont donc relativement éloignées d'une exploration et d'une vérification holistiques des théories sur les processus d'interprétation (on trouvera de nombreux articles illustrant la recherche empirique en interprétation de conférence dans Lambert et Moser-Mercer 1994, Tommola 1995, Danks et coll. 1997, Englund Dimitrova et Hyltenstam 2000, Garzone et Viezzi 2002, Pöchhacker et Shlesinger 2002, Pöchhacker 2004, ainsi que de mini-comptes rendus dans le CIRIN Bulletin). Il y a bien des vérifications de tel aspect ponctuel d'un modèle ou de telle incidence d'un principe ou d'une structure cognitive modélisée, mais on ne trouve dans la littérature aucune tentative de vérification complète d'un modèle de processus. La chose n'est d'ailleurs guère étonnante, dans la mesure où les modèles un tant soit peu élaborés, tels que celui de Moser ou celui de Setton, comportent des modules, étapes et interrelations internes multiples, dont la vérification demanderait des centaines d'études au bas mot, et les ressources institutionnelles, financières et humaines de la communauté des chercheurs en interprétation sont infinitésimales par rapport aux besoins. À la différence de la situation en traduction, il en résulte une séparation très nette dans la recherche sur l'interprétation de conférence entre la théorie sur les processus et la recherche empirique.

\section{Recherche sur les processus et enseignement}

\subsection{La recherche sur les processus apporte-t-elle de bonnes méthodes d'enseignement?}

\subsubsection{Les limites}

Jusqu'ici, les résultats des études sur le processus de la traduction et de l'interprétation se résument donc essentiellement à :

- Des observations de phénomènes (linguistiques, physiologiques, cognitifs) et de comportements (notamment les stratégies de traduction), surtout chez des étudiants, et en partie chez des professionnels.

- Des hypothèses et théories sur les raisons et mécanismes sous-jacents. Ces hypothèses et théories évoluent en suivant les théories des sciences cognitives, par transfert, mais ne sont validées directement par la recherche empirique sur des tâches de traduction et d'interprétation que dans une mesure très modeste, sur des éléments précis plutôt que sur des modèles globaux. Ainsi, quelques travaux intéressants sont 
à signaler sur la mémoire de travail chez les interprètes (Padilla 1995 et surtout Liu 2001), et Gile a pu obtenir, à l'appui de ses modèles d'Efforts, des résultats corroborant l'idée que les interprètes travaillent à proximité de la saturation cognitive (1999), que la prise de note chez les débutants consomme de l'attention au détriment de l'écoute (1991), ou que les différences observées dans les performances en simultanée et en consécutive sont conformes à des hypothèses dérivées de ces modèles (2001 - voir plus loin), mais n'a pas pu valider solidement l'ensemble de son modèle ou le faire progresser sur la base de vérifications empiriques.

En outre, les phénomènes et comportements observés sont le fruit d'opérations d'état des lieux, souvent avec des comparaisons entre étudiants et professionnels, mais ils ne sont que rarement les résultats d'expérimentations pédagogiques avec des méthodes ou des matériels. Autrement dit, si les chercheurs traductologues cherchent des indices sur les processus traductionnels chez des étudiants débutants, des étudiants avancés et des professionnels, ils n'en sont pas encore, dans l'ensemble, à tenter de mesurer l'impact de la mise en œuvre de nouvelles méthodes d'enseignement (ou de travail) sur la base de ces indications obtenues en matière de processus (voir Moser-Mercer 1997: 256).

Quant aux hypothèses et théories, certaines restent très éloignées des préoccupations traductionnelles pratiques. Ainsi, en matière d'interprétation de conférence, il est admis depuis longtemps qu'un aspect crucial de l'interprétation est le caractère limité des ressources attentionnelles disponibles. Ce facteur étant connu, les différences dans la conception de l'architecture de la mémoire de travail selon les modèles des uns et des autres (voir l'article de A. Mizuno dans ce numéro spécial) ont-elles une réelle incidence sur les paramètres pratiques de l'interprétation ou sur la manière de l'enseigner? Le modèle théorique de B. Moser (1978) apportait-t-il des idées pratiques nouvelles aux enseignants? Dans son article, elle propose un ensemble d'exercices (p.361-368) destinés à développer la capacité des étudiants à traiter des matériaux linguistiques, à maîtriser la simultanéité de la production du discours et de la compréhension, et à entraîner la mémoire (p. 361). Ces exercices sont certes utiles, mais la perception de leur utilité doit-elle vraiment passer par le niveau de détail de l'analyse proposée par Moser? Dès 1969, I. Pinter s'était intéressée à la capacité de parler et d'écouter en même temps, et avait montré que la pratique permettait de s'améliorer, sans recourir à des théories complexes. De même, R. Setton (1999) ne préconise pas dans son livre de méthodes de formation sur la base de son modèle. Bien au contraire, il marque avec lucidité et non sans humour ses réserves quant à l'idée de présenter aux étudiants les détails techniques des théories de la pragmatique ou les maximes de Grice, en se bornant à recommander la variété dans les discours proposés aux étudiants à titre d'entraînement (p.283). La psychologue Annette de Groot (2000) critique (p.54) le point de vue selon lequel l'acquisition des habilités cognitives complexes se fait au mieux par un entraînement majoritairement holistique, et cite des études suggérant que l'entraînement à des sous-tâches spécifiques peut être plus efficace. Si l'on adopte cette deuxième hypothèse, les modèles cognitifs élaborés dans le cadre du paradigme du traitement de l'information présentent un intérêt non négligeable en identifiant des modules sur lesquels la formation pourrait se concentrer pour optimiser les performances des étudiants. C'est peut-être l'inaccessibilité de ces modèles au commun des mortels qui est à l'origine de la réticence de la plupart des enseignants. Et pourtant, M. Shlesinger, interprète et enseignante qui a étudié le 
langage et les théories des cogniticiens, exprime des réserves quant à la validité écologique des études sur des facteurs isolés dans un contexte expérimental restreint par rapport à la tâche de l'interprète, qui dépend lourdement de nombreux facteurs contextuels et de leurs interactions (Shlesinger 2000). L'utilité des modèles cognitifs un tant soit peu élaborés pour suggérer et développer des méthodes de formation et d'entraînement pratiques est donc loin de faire l'unanimité, que ce soit parmi les formateurs-praticiens ou parmi les formateurs-théoriciens.

\subsubsection{Les obstacles}

Le caractère modeste des résultats concrets de la recherche sur les processus en matière d'interprétation de conférence s'explique essentiellement par deux ensembles de facteurs qui pèsent particulièrement lourd. L'un est la complexité des processus en question, du fait du grand nombre de facteurs potentiellement pertinents, dans le contenu et la forme des discours originaux, dans les conditions de travail, dans les caractéristiques linguistiques des langues concernées, dans les connaissances pertinentes des interprètes et dans leurs habiletés cognitives, etc. Cette multiplicité des facteurs et de leurs interactions est source d'une grande variabilité dans les performances, variabilité qui peut masquer les effets réels d'une variable donnée à moins que ces effets soient très importants. Ce phénomène est bien connu dans les milieux scientifiques et technologiques, mais peut-être est-il intéressant de le rappeler aux traductologues, qui ont parfois tendance à tirer des conclusions sur la base d'une seule étude sans chercher à éliminer la possibilité d'un effet fantôme dû à la variabilité.

Soit une étude qui recherche l'effet de l'expérience de l'interprète sur ses performances. Supposons qu'en «réalité», celles-ci s'améliorent très rapidement en début de parcours du fait d'un apprentissage initial des techniques et leur assimilation, et continuent à s'améliorer, mais de plus en plus lentement, au fil de la maturation des compétences et des connaissances de l'interprète. Supposons qu'il est possible de mesurer les performances de l'interprète sur une échelle de 0 à10, et que la courbe «réelle» de l'évolution correspond en moyenne aux valeurs suivantes (tableau 1 et figure 2):

TABLEAU 1

Progression «réelle» des performances de l'interprète au fil des ans

\begin{tabular}{|l|c|c|c|c|c|c|}
\hline Expérience en années & 0 & 5 & 10 & 15 & 20 & 25 \\
\hline Performances & 1 & 5 & 7 & 8 & 8,5 & 8,8 \\
\hline
\end{tabular}

FIGURE 2

Effet d'une variabilité aléatoire sur les performances de l'interprète

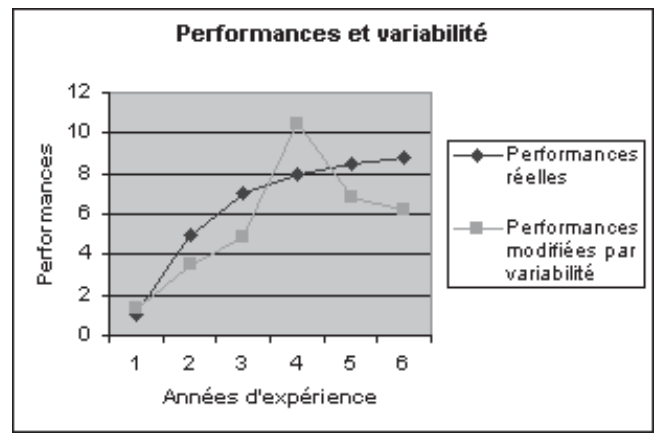


De nombreux auteurs mentionnent la difficulté de trouver des interprètes professionnels pour leurs études empiriques, et rares sont les travaux faits avec plus d'une dizaine de professionnels. Si l'on souhaite vérifier la progression des compétences des interprètes au fil de leur expérience sur des segments de 5 ans dans le cadre d'une étude empirique, il paraît difficile de recruter plus de 2 ou 3 sujets par segment (soit un total de 10 à 15 sujets). Étant donné sa taille, chaque groupe de 2 ou 3 interprètes peut présenter une grande variabilité dans l'accomplissement de la tâche expérimentale (le risque serait nettement plus faible si chaque groupe comportait une dizaine de personnes). Soit une variabilité aléatoire dans chacun de ces groupes allant de $+30 \%$ à $-30 \%$ par rapport à leurs performances réelles (dans la littérature, par exemple dans Lamberger-Felber, 1998, on trouve fréquemment une variabilité bien plus grande), par exemple selon la répartition suivante (tableau 2):

TABLEAU 2

Effet de la variabilité dans un échantillon donné sur les performances réelles de l'interprète

\begin{tabular}{|l|c|c|c|c|c|c|}
\hline Expérience en années & 0 & 5 & 10 & 15 & 20 & 25 \\
\hline Variabilité & $+30 \%$ & $-30 \%$ & $-30 \%$ & $+30 \%$ & $-20 \%$ & $-30 \%$ \\
\hline Performances modifiées par la variabilité & 1,3 & 3,5 & 4,9 & 10,4 & 6,8 & 6,16 \\
\hline
\end{tabular}

Le tableau 2 montre les performances qui pourraient être mesurées du fait de cette variabilité (voir aussi fig. 2). Au lieu de montrer une progression rapide qui se poursuit mais de moins en moins vite, elles suggèrent une progression régulière pendant 10 ans, suivie d'une accélération sur 5 ans, et surtout une baisse des performances après 15 ans d'expérience. Cet effet fantôme ne risque-t-il pas de conduire les chercheurs (et les employeurs!) sur une fausse piste?

Les effets de la variabilité aléatoire sur la détection du «signal» recherché peuvent être combattus par différents moyens, l'un étant une meilleure prise en main des procédures de recherche (questions, tâches et mesures plus précises), le deuxième étant le recours (difficile à mettre en œuvre dans la recherche sur l'interprétation) à des échantillons de plus grande taille, et le troisième étant les réplications.

Il existe un autre facteur perturbateur qui génère du «bruit» (des variations qui empêchent de capter le signal), à savoir l'influence de variables pertinentes qui ne sont pas prises en compte dans la conception de l'étude. Soit une étude de l'évolution de la compétence des interprètes au fil des ans qui ne tient pas compte du facteur «attitude». Il est plausible que l'attitude de l'interprète vis-à-vis de sa tâche influe sur ses performances, quel que soit son niveau de compétence. Supposons que l'attitude des jeunes interprètes est très positive, qu'elle subit une certaine érosion par la suite, et qu'elle se rétablit chez les interprètes expérimentés qui continuent à exercer leur métier après 20 ans de cabine, selon le tableau 3:

TABLEAU 3

Effet général de l'attitude sur les performances de l'interprète

\begin{tabular}{|l|c|c|c|c|c|c|}
\hline Expérience en années & 0 & 5 & 10 & 15 & 20 & 25 \\
\hline Effet de l'attitude & +++ & ++ & - & - & + & + \\
\hline
\end{tabular}


Supposons que cet effet général est relativement important (tableau 4):

TABLEAU 4

Effet important de l'attitude sur les performances de l'interprète

\begin{tabular}{|l|c|c|c|c|c|c|}
\hline Expérience en années & 0 & 5 & 10 & 15 & 20 & 25 \\
\hline Effet important de l'attitude & +3 & +2 & -3 & -1 & +1 & +1 \\
\hline Performances modifiées par l'attitude & 4 & 7 & 4 & 7 & 9,5 & 10,8 \\
\hline
\end{tabular}

Le tableau qui se dégage des résultats mesurés est alors assez différent de la réalité, puisque l'on a l'impression de fluctuations pendant les 15 premières années, et d'une hausse par la suite (fig.3). En revanche, si l'effet de l'attitude est faible par rapport aux autres variables, par exemple d'une amplitude 10 fois moins importante que celle postulée ci-dessus, on obtient les performances suivantes (tableau 5):

TABLEAU 5

Effet peu important de l'attitude sur les performances de l'interprète

\begin{tabular}{|l|c|c|c|c|c|c|}
\hline Expérience en années & 0 & 5 & 10 & 15 & 20 & 25 \\
\hline Effet peu important de l'attitude & $+0,3$ & $+0,2$ & $-0,3$ & $-0,1$ & $+0,1$ & $+0,1$ \\
\hline Performances modifiées par l'attitude & 1,3 & 5,2 & 6,7 & 7,9 & 8,6 & 8,9 \\
\hline
\end{tabular}

FIGURE 3

Effet de l'attitude sur les performances de l'interprète

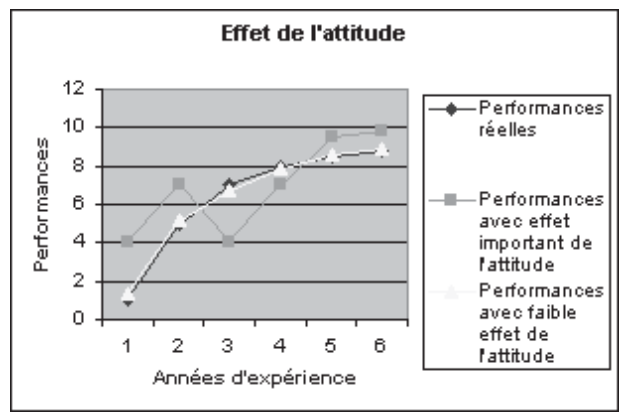

Dans un tel cas d'espèce, le fait de ne pas avoir pris en compte la variable attitude dans la conception de l'expérience (par exemple en interrogeant les interprètes làdessus avant de les sélectionner pour l'étude) n'a pas de retentissement important sur les résultats.

Si ces évidences sont rappelées ici, c'est que dans la recherche sur les processus, l'utilité des idées pour la formation dépend en grande partie de l'amplitude de l'effet des variables pertinentes. Quand cet effet est important, il peut apparaître clairement en dépit du «bruit» émanant de la variabilité et des variables parasites. Mais quand il est important, peut-être les formateurs n'ont-ils pas besoin de la recherche scientifique pour le détecter et en tirer des enseignements. Quand il l'est moins, seul un volume de recherche empirique important peut le mettre en évidence. 


\subsubsection{Perspectives}

Il est peu probable que la population des interprètes de conférence en exercice, qui est probablement de quelques milliers de personnes dans le monde, tous centres et toutes langues confondus, connaisse une forte explosion démographique au cours des décennies à venir. Il n'est pas plausible non plus qu'une importante infrastructure de recherche sur l'interprétation de conférence se développe pendant cette même période. Il ne semble donc pas réaliste de s'attendre à une masse de travaux sur des échantillons de taille suffisante pour que les modèles complexes puissent être validés ou évoluer sensiblement sur la base de travaux empiriques sur l'interprétation. Il faut plutôt s'attendre à ce que l'évolution théorique continue à s'appuyer sur les travaux des cogniticiens en dehors de l'interprétation, avec importation vers l'interprétation et des interrogations et incertitudes sur leur transférabilité.

Dans la traduction écrite, la recherche sur le processus semble plus fructueuse en matière de méthodes d'enseignement, peut-être parce qu'il s'agit de guider les étudiants vers des stratégies où les contraintes cognitives ne jouent qu'un rôle relativement faible, alors qu'en interprétation, le problème principal est celui de la gestion des ressources cognitives: les observations réalisées en matière de lecture de l'original, de consultation de sources, d'habitudes de relecture et d'auto-révision grâce aux protocoles de verbalisation à voix haute permettent de donner des conseils précis aux étudiants pour améliorer leurs méthodes de travail sans craindre que l'effet de telle composante cognitive du processus annule les bienfaits de ces suggestions. Ces possibilités vont probablement s'élargir avec la réintroduction dans la recherche de procédures de rétrospection, en traduction comme en interprétation (voir par exemple Ivanova, 2000, Mead 2002, Gile 2004). Pour les enseignants, ce type de recherche sur les processus, moins axé sur la théorie cognitive et plus focalisé sur les actes, stratégies et motivations n'est-il pas plus prometteur en matière d'applications directes à la formation?

\subsection{L’utilité explicative des théories sur le processus}

Quand on quitte les modèles de processus détaillés selon le paradigme du traitement de l'information pour passer à des conceptualisations plus holistiques, le tableau est quelque peu différent. Certes, les modèles holistiques tels que la théorie du sens de D. Seleskovitch ou les modèles d'Efforts de D. Gile n'ont pas en tant que tels vocation à évoluer comme les modèles cognitifs détaillés pour explorer de manière de plus en plus précise les différentes composantes du processus d'interprétation. En revanche, ils ont un rôle d'orientation bien plus clair. Ainsi, la théorie du sens, qui postule une déverbalisation entre compréhension et production et rejette l'idée d'une spécificité des processus en fonction de la paire de langues concernée, conduit à des orientations pratiques importantes : elle exclut les enseignements centrés sur les caractéristiques des langues concernées, et met l'accent sur l'analyse, sur la spontanéité dans l'expression, ce qui conduit naturellement à privilégier l'apprentissage de la consécutive. Les modèles d'Efforts sont plus spécifiques en ce qu'ils analysent l'interprétation en termes de charge cognitive et de ressources limitées pour des opérations de compréhension, de production du discours (ou de notes dans le cas de la première étape de la consécutive) et de gestion des ressources de la mémoire. À la différence de la théorie du sens, ils postulent la pertinence probable des spécificités linguistiques des 
langues de départ et d'arrivée (Gile 1995), donc la pertinence d'exercices spécifiques par langues. Ils considèrent également la prise de notes en consécutive comme une activité consommatrice de capacité de traitement et font donc des recommandations précises en matière de prise de notes.

En interprétation de conférence, l'élément provenant de la recherche (en l'occurrence de la recherche en psychologie cognitive) qui a la plus grande utilité pour les enseignants est celui de la limite des ressources cognitives de l'individu, avec une place particulière pour la mémoire de travail. Son pouvoir explicatif est très grand. Il intervient, en filigrane ou au premier plan, dans la quasi-totalité des modèles de processus conçus et développés au cours des 30 dernières années, et explique non seulement de nombreux problèmes et défaillances des interprètes, mais aussi, partiellement, leurs stratégies (qui sont également à base de normes, comme il est maintenant bien établi en traductologie, depuis que Gideon Toury a mis en évidence leur rôle dans la traduction). Les modèles d'Efforts, plus particulièrement, qui sont fondés sur cette idée de ressources cognitives limitées et sur «l'hypothèse de la corde raide» (Gile 1999), selon laquelle les interprètes travaillent en permanence à la limite de la saturation cognitive, sont largement utilisés pour expliquer aux étudiants la spécificité de l'interprétation par langues, les incidences du travail vers/à partir d'une langue maternelle/étrangère, les stratégies de prise de notes, les possibilités et limites de la consécutive par rapport à la simultanée (Gile 2001), etc. De petites expériencesdémonstrations de sensibilisation peuvent également être utilisées en classe. Par exemple, pour démontrer aux étudiants l'importance de la consommation de ressources attentionnelles de la prise de notes, Gile (1991) a divisé des classes d'élèvesinterprètes débutants en deux groupes pour l'interprétation consécutive d'un même discours, l'un des groupes étant autorisé à prendre des notes, et l'autre non. La comparaison de ce qu'ont retenu les uns et les autres est non seulement intéressante en tant que corroboration de l'hypothèse de la concurrentialité de l'écoute et de la prise de notes, mais aussi frappante pour les esprits des étudiants, qui acceptent plus volontiers l'idée de noter moins et d'écouter plus.

Curieusement, la traductologie des processus de la traduction écrite semble être moins focalisée sur la recherche d'explications pour les étudiants. Peut-être les raisons en sont-elles l'absence en traduction d'une pression cognitive aussi perturbatrice qu'en interprétation et une longue tradition d'orientation produit. Et pourtant, des opportunités existent. Ainsi, pour vérifier la légitimité d'une certaine liberté formelle dans la traduction, Gile réalise systématiquement en classe une expérience de verbalisation d'un énoncé qui démontre la variabilité inter- et intra-locuteurs, l'existence d'un facteur non délibéré dans l'énonciation et l'intrusion de différents types d'informations dans les messages informationnels (Gile 1995, 2005). D’après les évaluations écrites des cours recueillies chaque année, cette expérience semble efficace pour aider à libérer les étudiants débutants de leurs habitudes de traduction mot-à-mot acquises en cours de thème et de version.

\section{Conclusion}

Hormis l'utilisation explicative de la recherche sur le volet cognitif «profond» de la recherche sur les processus, les travaux concernés semblent pour l'instant avoir peu d'impact réel sur la pédagogie en traduction et interprétation. Compte tenu des 
ressources limitées dont dispose la communauté des chercheurs en interprétation, les réserves d'un grand nombre d'enseignants à l'égard de ce volet scientifique de la traductologie sont-elles justifiées? Certes, il appartient aux chercheurs au sein même de la communauté des chercheurs en interprétation de faire preuve de modestie quant aux applications de leurs travaux. D’un autre côté, la patience et la persévérance pourraient bien être payantes à long terme. Il n'est pas impossible que la recherche sur les processus finisse par isoler tel facteur de personnalité, telle habileté cognitive, telle action précise ayant un effet important et des applications didactiques directes, surtout si l'on continue à suivre l'évolution de la recherche cognitive extérieure à l'interprétation, où les ressources sont bien plus vastes. La survenue d'une découverte importante, parfois à un moment et en un lieu où on ne l'attend pas, est un scénario qui se répète souvent dans la recherche scientifique. Il semble raisonnable de continuer à s'intéresser à la recherche sur les processus, sans en attendre des retombées immédiates, mais en gardant l'esprit ouvert.

\section{RÉFÉRENCES}

ANDres, D. (2000): Konsekutivdolmetschen und Notation. Empirische Untersuchungen mentaler Prozesse bei Anfängern in der Dolmetscherausbildung und professionellen Dolmetschern, thèse de doctorat, Université de Vienne.

Cirin BUlLETIN <http://perso.wanadoo.fr/daniel.gile.>

Dam, H. (2004) : «Interpreters' Notes: On the Choice of Form and Language», in G. Hansen, K. Malmkjaer, and D. Gile, (eds.), Claims, Changes and Challenges in Translation Studies, Amsterdam/Philadelphia, John Benjamins, p. 251-261.

Danks, J., Shreve, G., Fountain, S., McBeath, M. (eds.) (1997): Cognitive Processes in Translation and Interpreting, Thousand Oaks, London and New Delhi, Sage Publications.

De Groот, A. (2000): "A Complex-skill Approach to Translation and Interpreting", in TIRKKONEN-CONDIT and JÄÄSKELÄINEN, p. 53-68.

Englund Dimitrova, B. and K. Hyltenstam (eds.) (2000): Language Processing and Simultaneous Interpreting, Amsterdam/Philadelphia, John Benjamins.

Fraser, Janet (2000): «What Do Real Translators Do? Developing the Use of TAPS from Professional Translators», in TirkKonEN-Condit and JäÄSKELÄINEN, p. 111-120.

Garzone, G. and M. Viezzi (eds.) (2002): Interpreting in the 21st Century, Amsterdam/Philadelphia, John Benjamins.

Gile, D. (1991): «Prise de notes et attention en début d'apprentissage de l'interprétation consécutive - une expérience démonstration de sensibilisation", Meta 36-2, p. 432-441.

Gile, D. (1995): Basic Concepts and Models for Interpreter and Translator Training, Amsterdam/ Philadelphia, John Benjamins.

Gile, D. (1999): «Testing the Effort Models' Tightrope Hypothesis in Simultaneous Interpreting - A Contribution», Hermes n ${ }^{\circ} 23$, p. 153-172.

Gile, D. (2001): «Consecutive vs. Simultaneous: Which Is more Accurate?», Interpretation Studies $\mathrm{n}^{\circ} 1$, p. 8-20.

Gile, D. (2004) : «Integrated Problem and Decision Reporting as a Translator Training Tool», The Journal of Specialised Translation $\mathrm{n}^{\circ}$ 2, p. 2-20, <www.jostrans.org $>$.

GiLe, D. (2005): La traduction. La comprendre, l'apprendre, Paris, PUF.

Gran, L. and J. Dodds (eds.) (1989): The Theoretical and Practical Aspects of Teaching Conference Interpretation, Udine, Campanotto Editore.

Hansen, G. (ed.) (1999): Probing the Process in Translation: Methods and Results, Copenhagen Studies in Language 24, Copenhagen, Samfundslitteratur.

Hansen, G. (ed.) (2003): Empirical Translation Studies: Process and Product. Copenhagen Studies in Language 27, Copenhagen, Samfundslitteratur. 
Herbert, J. (1952): Manuel de l'interprète, Genève, Georg.

Hönig, H. (1995): Konstruktives Übersetzen, Tübingen, Stauffenburg Verlag.

Ivanova, A. (2000): «The Use of Retrospection in Research on Simultaneous Interpreting», in TirkKonen-Condit and JäÄSKELÄInen, p. 27-52.

Kussmaul, P. (1995): Training the Translator, Amsterdam/Philadelphia, John Benjamins.

Lamberger-Felber, H. (1998): Der Einfluss kontextueller Faktoren auf das Simultandolmetschen. Eine Fallstudie am Beispiel gelesene Reden, unpublished doctoral dissertation, Karl Franzens Universität Graz.

Lambert, S. and B. Moser-Mercer (eds.) (1994): Bridging the Gap. Empirical Research in Simultaneous Interpretation, Amsterdam/Philadelphia, John Benjamins.

Liu, M. (2001): Expertise in Simultaneous Interpreting; A Working Memory Analysis, doctoral dissertation, University of Texas at Austin.

MacKintosh, J. (1983): Relay Interpretation: An Exploratory Study, unpublished MA dissertation, Birkbeck College, University of London.

Mead, P. (2002): Évolution des pauses dans l'apprentissage de l'interprétation consécutive, thèse de doctorat, université Lyon 2.

Moser, Barbara (1978) : «Simultaneous Interpretation: A Hypothetical Model and its Practical Application », in D. Gerver and W. Sinaiko (eds.), Language Interpretation and Communication, New York and London, Plenum Press, p. 353-368.

Moser-Mercer, B. (1997) : «The Expert-Novice Paradigm in Interpreting Research », in E. Fleischmann, W. Kutz and P.A. Sснмiтt (eds.), Translationsdidaktik: Grundfragen der Übersetzungswissenschaft, Tübingen, Gunter Narr, p. 255-261.

Padilla, P. (1995): Procesos de memoría y atención en la interpretación de lenguas, tesis doctoral, Universidad de Granada.

Paneth, E. (1957): An Investigation into Conference Interpreting, M.A. thesis, University of London.

Pinter, I. (1969): Der Einfluß der Übung und Konzentration auf simultanes Sprechen und Hören, thèse de doctorat, université de Vienne.

PöchHacker, F. (2004): Introducing Interpreting Studies, London and New York, Routledge.

Pöchhacker, F. and M. Shlesinger (eds.) (2002): The Interpreting Studies Reader, London and New York, Routledge.

Setton, R. (1999): Simultaneous Interpretation. A Cognitive-Pragmatic Analysis, Amsterdam/ Philadelphia, John Benjamins.

Setton, R. (2003): «Models of the Interpreting Process", in Á. Collados Ais and J. A. Sabio Pinilla (eds.) (2003): Avances en la investigación sobre interpretación, Granada, Comares, p. 29-89.

Shlesinger, M. (2000): «Interpreting as a Cognitive Process: How Can We Know What Really Happens?», in TirkKonen-Condit and Jä̈̈SKeläInen, p. 3-15.

Snell-Hornby, M., Nönig, H., Kussmaul, P. and P. Schmitt (Hrsg.) (1999): Handbuch Translation, Tübingen, Stauffenburg Verlag.

Tirkkonen-Condit, S. and R. JäÄskeläInen (eds.) (2000): Tapping and Mapping the Processes of Translation and Interpreting, Amsterdam/Philadelphia, John Benjamins.

Tommola, J. (ed.) (1995): Topics in Interpreting Research, Turku, University of Turku, Centre for Translation and Interpreting.

Van Hoof, H. (1962): Théorie et pratique de l’interprétation, München, Max Hueber Verlag. 